decrease in current amplitude. The authors further established that the late viral protein ICP34.5 is crucial for the effect of HSV-1 on sodium currents, as infection with viruses that lack such a protein did not result in sodium channel loss.

In addition to identifying a mechanism to account for the decrease in neuronal excitability that accompanies HSV-1 infections, the data of Storey et al. point to a new way to regulate the availability of sodium channels at the plasma membrane. What intracellular pathways does this process engage? Do neurons use this mechanism under physiological conditions? HSV-1 and, in particular, ICP34.5 will be useful tools to answer these questions.

$$
\text { Juan Carlos López }
$$

(D) References and links ORIGINAL RESEARCH PAPER Storey, N. et al. Selective internalization of sodium channels in rat dorsal root ganglion neurons infected with herpes simplex virus-1. J. Cell Biol. 158, 1251-1262 (2002)

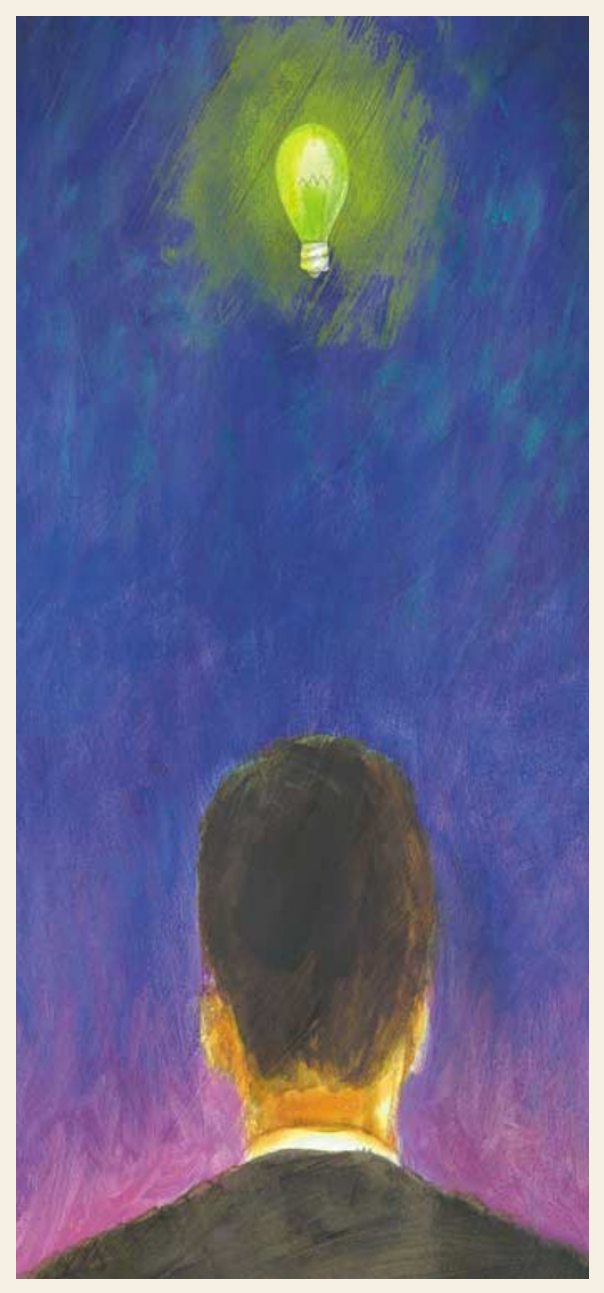

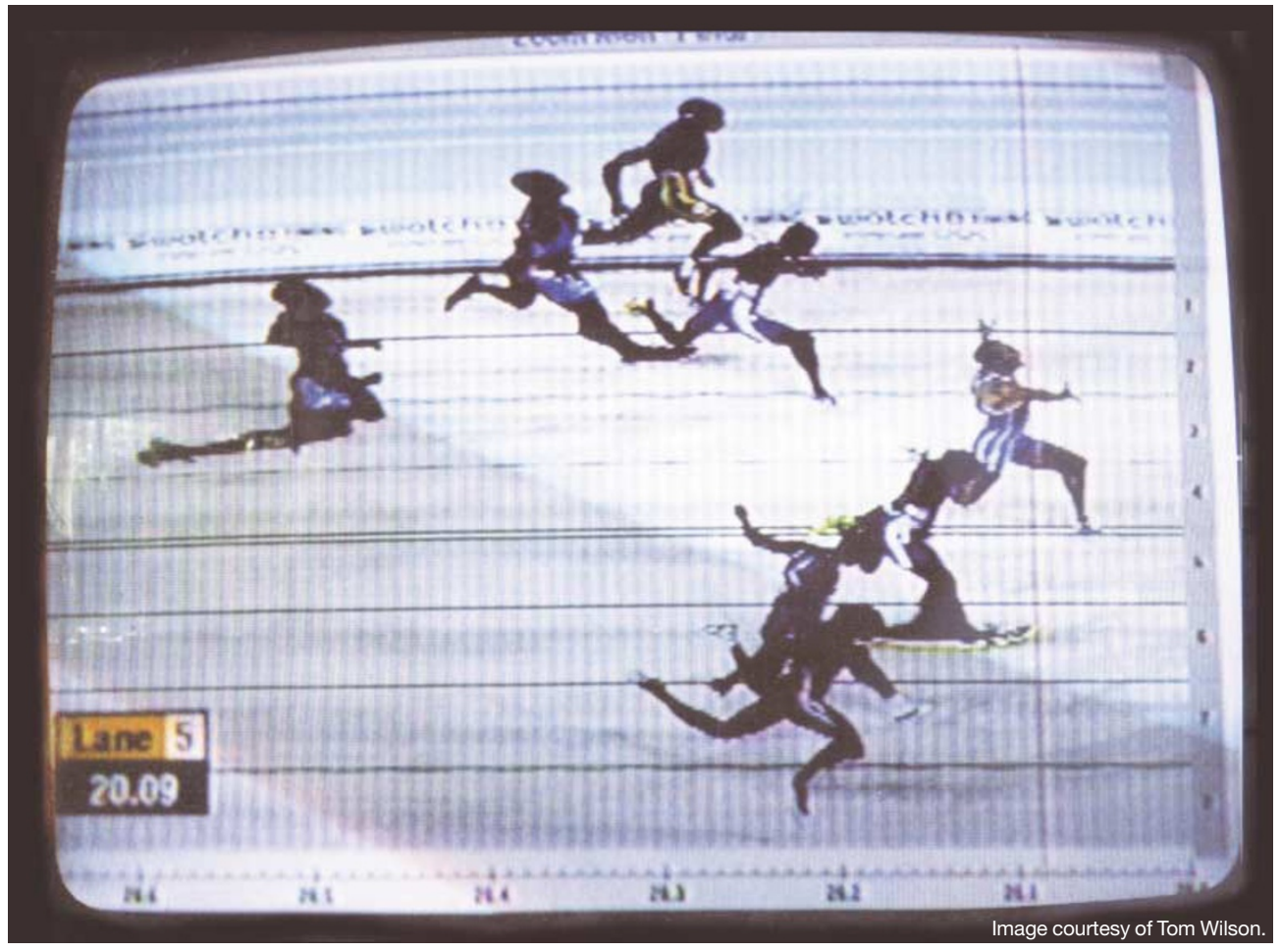

\section{LEARNING AND MEMORY}

\section{Action replay}

The fact that we are able to recall previous events in detail, even those from the distant past, indicates that we have a robust neural system for the acquisition and storage of memories. But despite this impressive storage capacity, in the minutes to days after an event, our memory of the experience is prone to disruption. Hoffman and McNaughton argue that this period of lability reflects the way in which new memories are converted into a longlasting form. Reporting in Science, they provide evidence that memory consolidation involves the reactivation of distributed components of 'memory traces' during periods of behavioural inactivity that follow an event.

According to the 'trace-reactivation theory' of memory consolidation, in 'offline' periods after an event - for example, during quiet waking or sleep - neurons in higher-level cortical regions are thought to activate cells in lower-level regions that were also active during the experience. Repeated co-activations of lower-level ensembles result in the formation of connections that are necessary to encode the memory trace. Previous studies have shown that patterns of cortical activity that accompany an experience can be triggered spontaneously during subsequent periods of rest. Hoffman and McNaughton set out to test a second prediction of the theory - that distributed components of the reactivated memory trace should appear concurrently within relevant cortical sites.

The authors implanted a 12-by-12 lattice of electrodes into each of four areas of the primate neocortex - posterior parietal cortex $(\mathrm{PP})$, motor cortex (M), somatosensory cortex (SS) and dorsal prefrontal cortex (PFC). They recorded simultaneously from multiple individual neurons during an initial period of rest (rest 1), a sequential reaching behaviour and a second period of rest (rest 2). Cell-pair firing-rate correlations were then calculated for each epoch. The authors found that, for PP-PP, M-M, SS-SS, PP-M, PP-SS and M-SS cell pairs, firing correlations during the task were more similar to those of rest 2 than to those of rest 1 . This indicates that neurons that were co-activated in these regions during the task tended to be activated together afterwards. No such pattern was seen for cell pairs that included neurons of the PFC. Hoffman and McNaughton went on to show that the 'temporal bias' of cell-pair interactions during the task was preserved in rest 2 in the $M$ and SS. So, if one cell tended to fire after another during the task period, then this firing sequence tended to recur in rest 2 . This preservation of temporal bias was not observed for PFC neurons.

These data lend support to the proposal that memory-trace reactivation occurs in a coordinated, distributed manner across the neocortex. Interestingly, no evidence was found of trace reactivation in the $\mathrm{PFC}$, which has been implicated in memory retrieval in humans. Further studies will be needed to elucidate the mechanisms that underlie memory-trace reactivation, and to establish whether or not the PFC has a part to play in this process.

Rebecca Craven

6) References and links ORIGINAL RESEARCH PAPER Hoffman, K. L. \& McNaughton, B. L. Coordinated reactivation of distributed memory traces in primate neocortex. Science 297, 2070-2073 (2002) FURTHER READING Qin, Y. L. et al. Memory reprocessing in FURTHER READING Qin, Y. L. et al. Memory reprocessing in
corticocortical and hippocampocortical neuronal ensembles. Phil. Trans R. Soc. Lond B 352, 1525-1533 (1997) | Kudrimoti, H. S. Reactivation of hippocampal cell assemblies: effects of behavioral state, experience, and EEG dynamics. J. Neurosci. 19, 4090-4101 (1999)

\section{WEB SITES}

Encyclopedia of Life Sciences: http://www.els.net amnesia | learning and memory 